\title{
Semiregular patterns on surfaces
}

\author{
Craig S. Kaplan* \\ Computer Graphics Lab, University of Waterloo
}

\begin{abstract}
A popular topic in computer graphics research is the synthesis of irregular textures on surfaces. However, very little work has considered how to decorate surfaces with ordered patterns. One exception is the work of Kaplan et al. [2004]. They map a periodic tiling onto a surface via the uniform domain of a geometry image. However, their technique suffers from significant distortion. Furthermore, the periodic tiling often is not compatible with the shape and boundary conditions of the geometry image, leaving awkward gaps that are stitched together with some difficulty.
\end{abstract}

These limitations can easily be overcome if we exploit more of the mathematical structure of patterns. Every periodic pattern in the plane belongs to one of 17 symmetry groups sometimes called wallpaper groups [Grünbaum and Shephard 1987]. When a pattern belongs to a group that contains $p 4$ as a subgroup, it can be seen as a tiling of the plane by copies of a decorated square. Note that the square's decoration has finite $c 4$ symmetry, and that its vertices are also centres of rotational symmetry of the entire pattern.

If we now drop the constraint that four squares meet around every vertex, we can map this fragment of the pattern onto any edge-toedge arrangement of squares in $2 \mathrm{D}$ or $3 \mathrm{D}$ in a way that preserves most of the pattern's structure. In particular, the pattern fits conveniently into the parameter domains of a semi-regular surface parameterization by squares. Thus any planar pattern of symmetry type $p 4, p 4 g$ or $p 4 m$ (the three groups with $p 4$ as a subgroup) can be mapped seamlessly onto a suitably parameterized surface. A similar analysis shows that patterns of type $p 6$ and $p 6 m$ (which contain $p 6$ as a subgroup) map seamlessly onto surfaces with semiregular edge-to-edge parameterizations by equilateral triangles.

Because of recent advances in mesh parameterization, this technique has a straightforward implementation, illustrated in Figure 1. We use Spectral Surface Quadrangulation [Dong et al. 2006] for square domains and Globally Smooth Parameterization [Khodakovsky et al. 2003] for triangles. Parameterized meshes can simply be texture mapped with copies of a single square or triangular swatch from a pattern belonging to one of the five wallpaper groups discussed above.

The restriction to five of the 17 wallpaper groups is a minor one. A large fraction of historical and contemporary ornament belongs to one of these groups. All but one of the examples in the paper by Kaplan et al. belong to $p 4 m$ or $p 6 m$, and could therefore be rendered with lower distortion using this technique.

Figure 1 shows an example based on wallpaper group $p 4 m$. Examples for the remaining groups are shown in Figure 2.

\section{References}

Dong, S., Bremer, P.-T., Garland, M., Pascucci, V., AND HART, J. C. 2006. Spectral surface quadrangulation. ACM Transactions on Graphics (Proceedings of SIGGRAPH 2006) 25, 3, 1057-1066.

Grünbaum, B., And Shephard, G. C. 1987. Tilings and Patterns. W. H. Freeman.

\footnotetext{
*e-mail: csk@cgl.uwaterloo.ca
}

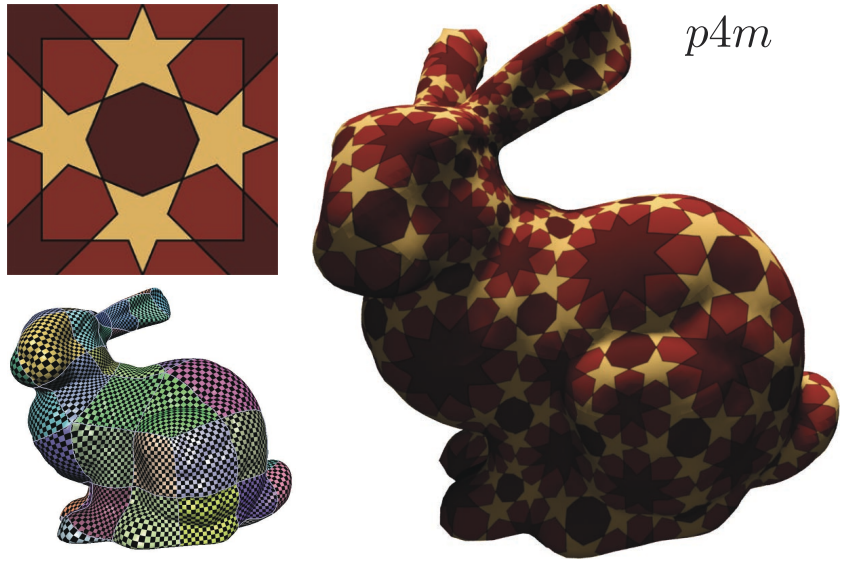

Figure 1: A demonstration of the mapping of a portion of an Islamic star pattern with $\mathrm{p} 4 \mathrm{~m}$ symmetry (top left) onto a parameterized mesh (bottom left).
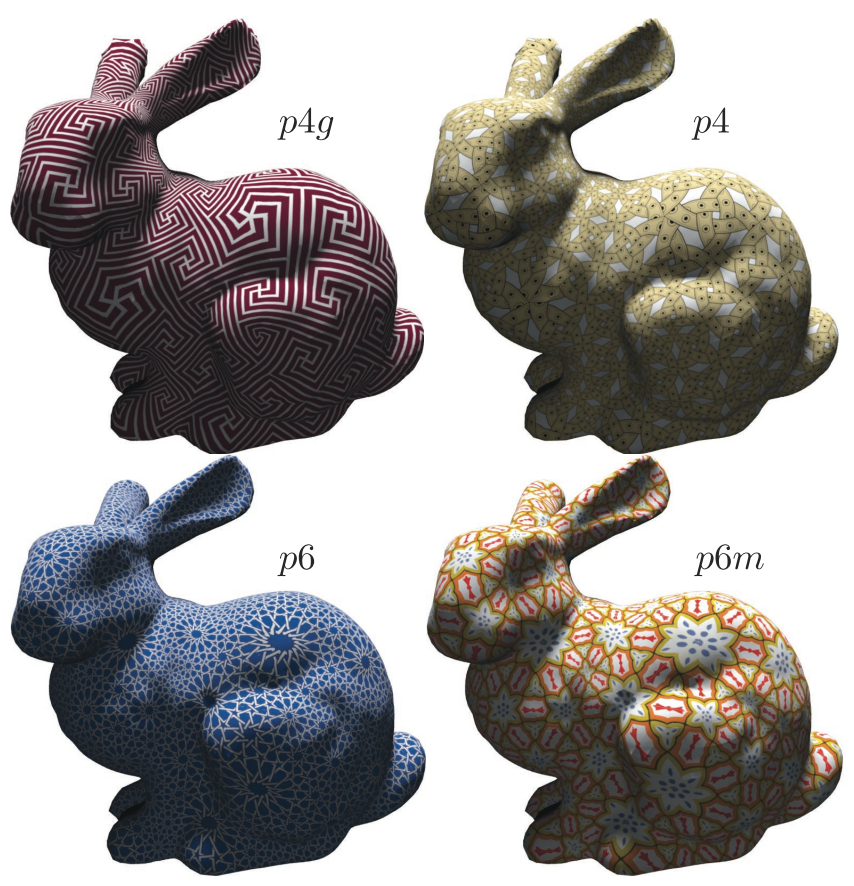

Figure 2: More examples produced with this technique, demonstrating the remaining wallpaper groups to which it applies.

Kaplan, M., Praun, E., And Cohen, E. 2004. Pattern oriented remeshing for Celtic decoration. In Proceedings of the 12th Pacific Conference on Computer Graphics and Applications (PG 2004), 199-206.

Khodakovsky, A., LiTKe, N., AND SCHRÖDER, P. 2003. Globally smooth parameterizations with low distortion. ACM Transactions on Graphics (Proceedings of SIGGRAPH 2003) 22, 3 (July), 350-357. 